\title{
CAPITAL SOCIAL Y DESARROLLO RURAL: REVISIÓN DE LOS APORTES EUROPEOS EN INVESTIGACIÓN APLICADA ${ }^{1}$
}

\section{SOCIAL CAPITAL AND RURAL DEVELOPMENT: REVIEW OF EUROPEAN CONTRIBUTIONS IN APPLIED RESEARCH}

Fecha recepción: 25 de noviembre 2018 / fecha aceptación: 17 de enero 2019

\section{Elena Pisani² y Stefano Micheletti ${ }^{3}$}

\section{Cómo citar este artículo:}

Pisani, E., y Micheletti, S. (2018). Capital social y desarrollo rural: revisión de los aportes europeos en investigación aplicada. Revista Pensamiento y Acción Interdisciplinaria, 4(2), 44-59. http://doi.org/10.29035/pai.4.2.44

\section{Resumen}

Este ensayo es uno de los productos de la colaboración entre la Facultad de Ciencias Sociales y Económicas de la Universidad Católica del Maule y el "Dipartimento Territorio e Sistemi Agro-Forestali" de la Universidad de Padua (Italia), gracias al apoyo financiero de CONICYT, a través del Concurso de Atracción de Capital Humano Avanzado del Extranjero (MEC). Uno de los objetivos del proyecto fue proponer un análisis de la literatura científica que trata el desarrollo local desde una perspectiva social y económica. Específicamente, en este trabajo hemos observado cómo se aborda el concepto de capital social en el desarrollo local, tanto a nivel internacional como en el marco europeo, proponiendo algunas reflexiones para el contexto chileno, aunque reconociendo las significativas diferencias.

Palabras claves: Capital social; Desarrollo rural; Investigación aplicada; Territorio; Redes sociales.

\footnotetext{
${ }^{1}$ Este ensayo corresponde a una versión sintetizada y actualizada del primer capítulo del libro "La misurazione del capitale sociale nei territori rurali" de Elena Pisani.

${ }^{2}$ Italiana, Titulada en Ciencias Políticas, PhD. en Land and Regional Economics. Académica del Departamento Territorio e Sistemi Agro-Forestali (TESAF) de la Universitá degli Studi di Padova, Italia. Email: elena.pisani@ unipd.it

${ }^{3}$ Italiano, Titulado en Ciencias Forestales y Ambientales. Mg. en Cooperación para el Desarrollo y Responsabilidad Social y Ambiental. Investigador del Centro de Estudios Urbano-Territoriales y académico de la Escuela de Sociología de la Universidad Católica del Maule, Chile. Email: smicheletti@ucm.cl
} 
Abstract

This work is one of the products of the collaboration between the "Faculty of Social and Economic Sciences" of the Catholic University of Maule (Chile) and the "Dipartimento Territorio e Sistema Agro-Forestale" of the University of Padova (Italy), thanks to the financial support of CONICYT- "Concurso de Atracción de Capital Humano Avanzado del Extranjero" (MEC). One of the central objective of the project was to propose an analysis of the scientific literature dealing with local development from a social and economic perspective. Specifically we have observed how social capital is contextualized in local development at international level and in the European context in order to propose possible reflections for the Chilean context, although acknowledging the significant differences.

Keywords: Social capital; Rural development; Applied research; Territory; Social networks.

\section{Las teorías del capital social}

El debate científico sobre capital social emerge con particular énfasis y vivacidad intelectual al final de los años ochenta. De todos modos, hay que hacer notar que varios sociólogos se refirieron a este concepto-aunque de manera implícita- desde la primera mitad del siglo XIX. Esto no debe sorprender, ya que como señalan Welzel, Inglehart, y Deutsch (2005), la investigación sobre capital social trata de dar respuesta a preguntas que todas las sociedades tienen en común, es decir: ¿qué lleva a todos los individuos a actuar según objetivos colectivos? Y sobre todo ¿qué mantiene unidas las sociedades?

Para las ciencias económicas, el interés hacia el capital social no está orientado solamente por la perspectiva positivista, sino también por la normativa. En efecto, los aspectos innovadores que han dado impulso a la investigación económica sobre el tema se relacionan en parte con el análisis de los factores inmateriales que pueden sostener el desarrollo, induciendo una mayor eficiencia y eficacia de los sistemas socio-económicos; por otro lado, se refieren al análisis de los aspectos positivos que el capital social podría vehicular en el sistema socioeconómico, con el fin de reducir situaciones de marginalidad y escasa integración socio-económica y las consiguientes indicaciones de policy.

El concepto de capital social aparece como tema transversal, aunque de forma no totalmente explícita, en los textos clásicos de la teoría sociológica. Emerge así en las investigaciones relativas a la clase y la solidaridad de clase de Marx y Engels, al espíritu público y la democracia de Toqueville, a la solidaridad y división del trabajo de Durkheim y, obviamente, en el análisis de las comunidades religiosas y el poder realizado por Weber. Sin embargo, es en la obra "The Rural School Community Center" de Lyd Hanifan (1916) donde se identifica la primera referencia explícita a este concepto, según su significado más moderno. El autor 
releva la importancia de la comunidad para el éxito de los institutos públicos de formación, junto con la participación comunitaria, para elevar la performance escolar de los estudiantes. Hanifan (1916) define el capital social como

aquellos elementos intangibles que cuentan más que cualquier otra cosa en la vida cotidiana de las personas: la buena voluntad, la amistad, la simpatía, la participación y las relaciones sociales entre quienes constituyen una unidad social. Si una persona entra en contacto con sus vecinos, y éstos con otros vecinos, se determina una acumulación de capital social que puede satis-facer inmediatamente las necesidades sociales individuales y que puede aportar una potencialidad social suficiente para el sustancial mejoramiento de las condiciones de vida de toda la comunidad. (p. 130).

Esta interpretación encierra sin duda los elementos cruciales de las interpretaciones posteriores, aunque en su tiempo no concitó el suficiente interés entre la comunidad científica. Algunas décadas más tarde, el concepto fue retomado en los estudios urbanísticos. En 1961, Jane Jacobs critica en sus trabajos la crisis de las grandes ciudades americanas y el modelo de desarrollo de la urbanidad moderna, sosteniendo la necesidad de recuperación de los núcleos urbanos y enfatizando el rol de la calle, del distrito, de la manzana, del barrio, de la cercanía, de la densidad y heterogeneidad de los edificios. Jacobs registra de hecho como en los barrios-dormitorios -construidos sin tener en cuenta los aspectos informales de las estructuras de las relaciones sociales- los efectos perversos de la actuación económica determinaron la pérdida de la capacidad de auto-regulación en sociedades altamente organizadas.

Desde la primera definición de capital social se ha recorrido mucho camino. El análisis de las determinantes de la agregación social no pertenece ahora solamente a la línea sociológica, sino que supera el límite disciplinario y entra al campo de los estudios politológicos y económicos, aunque con diferentes objetivos. En el primer caso, se busca especificar el rol normativo de las instituciones públicas sobre el capital social y, eventualmente, evidenciar los posibles trade off entre capital social y capital institucional. En el segundo caso, el capital social se ha considerado como un factor inmaterial de la producción, inserto en la estructura teórica de referencia del enfoque neoclásico. En consecuencia, los numerosos investigadores -según el ámbito disciplinar específico de pertenencia- han definido el concepto de capital social con diversas, y muchas veces contrapuestas, claves de lectura. 
En la dimensión sociológica el capital social es analizado a través de las redes de relaciones interpersonales. A tal propósito, algunos de los mayores referentes teóricos son: a) la teoría de las trayectorias sociales de Bourdieu (1980, 1986); b) la teoría de la elección racional de Coleman (1988, 1990); c) la teoría del embeddedness de las relaciones sociales en las acciones económicas de Granovetter (1973, 1985, 2018); y d) la más reciente teoría de network de Lin crea un puente teórico entre la sociología y la economía (1999, 2001, 2003, 2005, 2017).

Por otro lado, la perspectiva politológica sobre el capital social se vincula esencialmente con los estudios realizados por Putnam, Leonardi, y Nanetti (1993); allí se sostiene que el capital social puede ser definido como "la confianza, las normas de reciprocidad que regulan la convivencia y las redes de asociacionismo cívico, elementos que mejoran la eficiencia de la organización social, facilitando la coordinación de las acciones individuales y promoviendo iniciativas tomadas de común acuerdo” (p. 196).

En el ámbito de la microeconomía, los estudios sobre capital social tienden a reforzar la ortodoxia dominante, es decir la teoría neo-clásica, utilizando como una variable más del modelo. En esta línea trabaja Gary Becker (1974a, 1974b, 1996; Becker y Murphy, 2000), que en su teoría sobre las interacciones sociales tiende a explicar cada aspecto de la vida social (enamoramiento, matrimonio, divorcio, fe religiosa, abusos sexuales, etc.) como el resultado de la conducta de un sujeto económico racional y perfectamente informado. De todos modos, el concepto de capital social transfiere a la ciencia microeconómica una importante característica de la sociología: la característica del actor económico como sujeto socializado, cuya conducta puede ser influenciada por las relaciones entre individuos.

Finalmente, en el campo de la macroeconomía es cada vez más nutrida la literatura que estudia el rol del capital social en el crecimiento del PIB y su capacidad interpretativa acerca de las diferencias de renta per cápita entre naciones y en su interior. Como sostiene el premio Nobel para la economía Kenneth Arrow (1972) es posible argumentar que muchos de los atrasos económicos mundiales son posibles de explicar en función de la falta de confianza mutua. Esta aseveración, sin embargo, ha sido refutada por Bagnasco, Piselli, Pizzorno, y Trigilia (2001) según quienes no es posible definir a priori el efecto de la confianza y el capital social sobre el desarrollo económico. Solamente un análisis detallado e históricamente orientado puede ayudar a clarificar cómo variables de tipo cultural, político y económico -interactuando entre ellas- no solamente favorecen u obstaculizan el capital social, sino también condicionan las consecuencias de su uso en el desarrollo local; así, no puede considerarse de manera automática el capital social como un recurso para el desarrollo. Justamente por la incertidumbre del papel jugado por el capital social en el crecimiento económico, se están buscando metodologías para estimar su efectiva contribución. 
En síntesis, hasta el día de hoy no existe una interpretación unívoca del concepto. El capital social representa, entonces, una especie de laboratorio experimental de ideas particularmente estimulantes, más que un corpus teórico claramente codificado.

\section{Capital social y desarrollo rural: la perspectiva europea}

Hasta el momento, hemos introducido el concepto de capital social esencialmente desde el punto de vista teórico; la investigación aplicada es variada, aunque no abunda la producción bibliográfica vinculada a las relaciones entre capital social y desarrollo socio-económico de las áreas rurales y de los territorios locales, que es el eje central de este capítulo. En este apartado, se propone un breve análisis crítico de la literatura europea, y posteriormente un análisis de las contribuciones italianas.

\section{La producción científica europea}

En el ámbito europeo la relación entre capital social y desarrollo rural ha sido analizada sobre todo en contexto de áreas rurales caracterizadas por condiciones de marginalidad socio-económica. Al respecto, podemos mencionar el estudio de la Comisión Internacional para la Protección de los Alpes (CIPRA), realizado por Wiesinger, Vihinen y Tapio-Bistrom (2005) y ligado al proyecto "Futuro de los Alpes", que enfatizó el rol del capital y de la cohesión social para el éxito de las políticas de desarrollo rural. En particular, han sido objeto de estudio las áreas agrícolas que habían reportado buenos resultados en la reducción o contención de los fenómenos de degradación ambiental y de marginalización socio-económica; esto, con el objetivo de identificar buenas prácticas que pudieran replicarse, con oportunas adaptaciones, también en ámbitos territoriales con situaciones de debilidad estructural.

Otra publicación que trata más ampliamente el tema es el posterior trabajo de Wiesinger (2007), que propone un modelo interpretativo de las relaciones entre capital social, desarrollo rural y sistemas de governance. También en este caso, el foco está puesto en las áreas rurales débiles, sosteniendo la idea que la situación de marginalidad no se debe solamente a condiciones desfavorables o a recursos faltantes; para Wiesinger, existe entonces un factor intangible que contribuye a determinar la condición de marginalidad, una especia de missing link que según el autor es justamente el capital social. En esta interpretación, el capital social se basa en el potencial endógeno de las regiones. 
En el ámbito de esta línea de pensamiento, se ponen por ejemplo importantes programas como LEADER e INTERREG, que implícitamente estimulan el capital social y la capacidad de organización de la sociedad. En especial, el trabajo de Wiesinger evidencia que:

1) Las políticas que influencian las áreas rurales son diversificadas (agrícolas, rurales, ambientales, sociales, culturales, turísticas) y prevén una gama amplia de instrumentos asociados;

2) La marginalización puede definirse haciendo referencia a múltiples aspectos y puede ser monitoreada a través del uso de indicadores;

3) La relación entre políticas y capital social es representada por la governance, definida como la situación en que las políticas son cada vez más delineadas e implementadas en cooperación entre el sector público, el mercado y los actores de la sociedad civil;

4) La percepción de la sociedad civil sobre su propio territorio puede influenciar la dotación de capital social, pero puede ser verdadera también la relación inversa;

5) El capital social puede ser visto como un posible remedio, a nivel local, al retiro progresivo del Estado, es decir la sociedad civil organizada sustituye el actor gubernamental en la entrega de servicios a favor de la colectividad local.

Analizando el modelo de Wiesinger, se observa la existencia de dos posibles (y no excluyentes) esquemas de intervención del actor público para las áreas rurales: a través de la definición de políticas sectoriales o integradas, monitoreadas con la ayuda de indicadores, o bien a través de la transición desde sistemas de gobierno a sistemas de governance, donde la concertación de actores es la característica dominante y el trait d'union entre government y governance es representado justamente por el capital social existente a nivel local. Este último, se vincula entonces con el análisis del desarrollo rural sobre todo en relación con las modalidades de su activación a través del uso de idóneos instrumentos de governance. A nivel de políticas, es por lo tanto muy importante entender cuáles son los mecanismos que activan el capital social local, aspecto que necesariamente tiene que profundizarse a nivel de investigación.

Con respecto al principal instrumento de intervención en las áreas rurales definido a nivel de la Unión Europea a través de la Política Agrícola Comunitaria (PAC), se observa la aplicación de diferentes sistemas de government y governance; aparece particularmente interesante, en el segundo caso, el Programa LEADER, que se orienta hacia la lógica de activar, estimular o utilizar el capital 
social existente a nivel local, para la definición y actuación concertada de las estrategias de desarrollo rural. En este sentido, el fortalecimiento del capital social se expresa tanto en la dimensión estructural (entendida como identificación de nuevas estructuras organizativas que prevén la participación de actores locales o el mejoramiento de los instrumentos de planificación y de las consecuentes estructuras en una lógica de mayor participación), como en la normativa (entendida como norma social de comportamiento basada en la confianza y reciprocidad) y en la cognitiva (aprendizaje colectivo generado por las interacciones sociales en el territorio). La importancia para las áreas rurales europeas del Programa LEADER ha sido analizada también por Farrel e Thirion (2005), quienes profundizaron la reflexión acerca del capital social (de tipo estructural y cognitivo) como pre-condición para la activación de sistemas efectivos de governance del desarrollo rural.

\section{El contexto italiano}

Si nos concentramos en el ámbito de la investigación italiana, los principales temas explorados hasta ahora por los investigadores han sido: a) desarrollo rural, capital social y análisis de los sistemas territoriales locales (Cecchi et al., 2008), b) capital social e innovación (De Devitiis, Lopolito, Maietta, y Sisto, 2009), y c) capital social y análisis de redes sociales (Nardone, Sisto, y Lopolito, 2007b; Franceschetti, 2009; Pisani, Franceschetti, Secco, y Christoforou, 2017). Cabe destacar que estas grandes vertientes temáticas no copan totalmente el campo bibliográfico italiano, sin embargo, representan las principales discusiones que se están desarrollando en el ámbito de la economía agraria y sobre todo rural.

En relación con la perspectiva micro, meso y macroeconómica, los temas emergentes son: a) análisis del capital social individual de la empresa agrícola (escala micro), b) análisis de las relaciones entre capital social y sistemas territoriales locales que, según una parte importante de la bibliografía en el ámbito sociológico, aparece como la dimensión territorial más oportuna para el estudio del tema (escala mes), y c) análisis del capital social como indicador de la eficacia de los programas que tienen como objetivo el desarrollo de las áreas rurales según enfoques de tipo integrado (escala macro).

El análisis macroeconómico del capital social parece, por el momento, haberse desarrollado de manera incipiente en el ámbito de la ruralidad, más allá de algunas contribuciones descriptivas. Estas limitaciones son explicables debido a la falta de indicadores adecuados, pero existe también otro factor crítico: la dificultad -puesta en evidencia por la escuela sociológica- de analizar el capital social a un nivel demasiado amplio de agregación territorial. En otras palabras, debatir sobre capital social a escala nacional no garantiza una clara representación del fenómeno, sobretodo en contextos -como el italiano- donde existe mucha heterogeneidad territorial. 
No obstante, ya existen metodologías analíticas como las propuestas por el World Value Survey. Sin duda, estas no tienen como objetivo cuantificar de forma incontrovertible una estimación del valor del capital social en un determinado ámbito nacional o regional, pero pueden resultar de utilidad para generar comparaciones a nivel internacional.

Cabe destacar, de todos modos, que este tipo de análisis tienen como población objetivo esencialmente la población urbana, y la rural no es adecuadamente representada. Se abre entonces un campo muy amplio para los investigadores que se ocupan del desarrollo socio-económico de las áreas rurales, con el fin de comprender el rol del capital social -junto a las otras formas de capital- en la activación de los procesos de desarrollo, o bien mantener un territorio en condiciones de marginalidad o involución socio-económica.

En este sentido, es interesante el análisis propuesto por Cecchi et al. (2008) que conectan -en relación con las áreas rurales-estos temas: a) conocimiento contextual (o conocimiento no codificado), b) sistemas territoriales locales, partiendo por la literatura sobre distritos industriales, y c) capital social como factor que conecta los primeros dos elementos, con el efecto de reforzar la percepción del territorio como unidad homogénea de individuos que reconocen especificidad normas sociales y de reciprocidad, basadas en relaciones de confianza.

Este último aspecto evidencia la importancia de la cohesión social como factor de facilitación en la transmisión del conocimiento y en la difusión de innovaciones (sobre todo de carácter incremental), siempre que exista una propensión relevante a la innovación por parte de los sujetos económicos presentes en el territorio. En el trabajo de Cecchi et al. (2008), el nivel de análisis considerado más pertinente acerca de la relación entre áreas rurales y capital social es el mesoeconómico, es decir de los sistemas territoriales locales. Estas hipótesis fueron comprobadas en el sector de la Maremma (Región de Toscana), y en diversos sectores rurales europeos, sosteniendo que el capital social y el conocimiento contextual están fuertemente ligados en aquellos territorios caracterizados por una fuerte visión de identificación comunitaria, que transforma el área rural en una especie de distrito rural.

La conexión entre capital social y conocimiento contextual es desarrollada también por Vespasiano y Martini (2008), aunque sus áreas de análisis no fueron específicamente las rurales, sino más bien la adopción de innovaciones -en un sentido amplio- por parte de las empresas. Los autores evidencias que en el proceso de innovación empresarial el capital cognitivo del territorio -conformado por capital relacional, cultura internalizada y capital social- asume un rol relevante. Con respecto al capital cognitivo, los sistemas productivos locales disponen de factores invisibles (embedded) que son compartidos en el territorio, cuya materialización contribuye a determinar las características peculiares de cada territorio. Estos caracteres se han sintetizado a menudo bajo el concepto 
de milieu -específico y no transferible a otros ámbitos locales- y que se sostiene en: a) economías externas de carácter ambiental, b) cooperación en un marco de relaciones sociales estables reguladas por la confianza, c) historia, cultura y espíritu comunitario compartidos, y d) conocimientos compartidos. El conjunto de los conocimientos y saberes de un territorio, desde el conocimiento codificado al localizado (tácito), determina la economía cultural de un territorio local, y en consecuencia su potencial ventaja en el mercado global.

El análisis de Vespasiano y Martini (2008), focalizado en la relación entre innovación y capital social, ha sido posteriormente retomada por De Devitiis et al. (2009), ya no desde la perspectiva de la economía territorial, sino de la economía del sector de la agricultura biológica. Los autores hipotetizan que el capital social sea una de las mayores determinantes de la innovación en el sector biológico, particularmente sensible a la investigación y transferencia de innovaciones y usualmente caracterizado por pequeñas y medianas empresas que deben necesariamente recurrir a recursos externos -también de tipo relacional-para poder realizar sus inversiones. Basándose en un modelo probit, los autores confirman las hipótesis iniciales, ratificando los hallazgos de los estudios anteriores de Landry, Amara, y Lamari (2002) y de Subramaniam y Youndt (2005); éstos, demostraron que los incrementos marginales en el capital social estructural contribuyen, más que otras variables, al aumento de la capacidad de innovar de la empresa.

El tema de las relaciones entre capital social y desarrollo local rural ha sido analizado también por Franceschetti (2009); específicamente, su objetivo consistió en analizar el capital social estructural que se creó en el marco de los Grupos de Acción Local (GAL) ${ }^{4}$ de la Región Veneto, identificando una metodología que puede ser replicada también en otras regiones italianas. Franceschetti parte desde el estudio de Nardone, Sisto, y Lopolito (2007a), que presenta el coeficiente de densidad reticular aplicado en algunos GAL y definido como complemento al Coeficiente de Gini. El aporte innovador de Franceschetti se refiere al perfeccionamiento del coeficiente de densidad reticular en relación con estos dos aspectos:

1) Transitar desde categorías institucionales a categorías funcionales (diferenciadas sobre la base del sector económico de pertenencia);

2) Poner particular atención a las categorías institucionales críticas para los fines del coeficiente de densidad.

El nuevo coeficiente así determinado (y definido como coeficiente de diversificación funcional-reticular) evidencia entones la mayor o menor capacidad de los GAL de generar capital social estructural. En consecuencia, podría utilizarse también en los sistemas de monitoreo y evaluación de las iniciativas de desarrollo

\footnotetext{
${ }^{4}$ Unidades locales de planificación y gestión del programa LEADER de la Unión Europea.
} 
rural que utiliza en Programa LEADER. Una importante novedad en el trabajo de Franceschetti es la aplicación de la Social Network Anaysis (SNA) en los GAL, con el fin de identificar los clásicos indicadores de centralidad (degree centrality, closeness centrality, betweeness centrality, eigenvector centrality), evidenciando forma y dimensiones del capital social estructural.

Finalmente, el trabajo más reciente a nivel de análisis aplicado del capital social en contextos rurales ha sido desarrollado por Pisani et al. (2017); los autores propusieron una visión basada en la sinergia del capital social (Woolcock y Narayan, 2000), donde las instituciones públicas desempeñan un papel importante en la generación de un contexto social y político que permita una amplia cooperación y participación. Esto, promoviendo los derechos de las personas y proporcionando marcos legales para actuar colectivamente; pero también asegurando el diálogo público, la gobernabilidad democrática, la rendición de cuentas y la transparencia, a la vez de coordinar las relaciones entre los actores locales. Estas acciones, en conjunto, permiten la movilización y el trabajo conjunto para impulsar procesos de desarrollo local.

Cabe destacar que, en los últimos años, el papel del capital social en el desarrollo de las áreas locales y rurales ha recibido una atención cada vez mayor (e.g. Horlings y Marsden, 2012; Koutsou, Ragkos, y Partalidou, 2014; Tamásy y Revilla, 2016; Raagmaa, 2015). Diversos autores argumentan que éste les otorga a los actores locales la capacidad de limitar los impactos negativos del despoblamiento rural, el agotamiento de la biodiversidad, el desempleo y la exclusión social, así como apoyar la difusión de nuevos servicios, la innovación social, la protección del patrimonio natural y cultural y la creación de nuevas formas de gobernanza y gestión del paisaje (véase Farrell y Thirion, 2005; Wiesinger, 2007; Bosworth et al., 2016).

Considerando las ambigüedades conceptuales y los problemas de medición (véase Fine, 2001; Durlauf, 2002; Durlauf y Fafchamps, 2004; Teilmann, 2012), la evaluación del capital social se ha vuelto un tema central. Las principales críticas, en este sentido, apuntan a que las definiciones y medidas del concepto confunden a menudo insumos (redes, normas) con productos (formas de comportamiento cooperativo como la participación política y las organizaciones sociales); en algunos casos, además, se asumen resultados positivos en los territorios, desconociendo los impactos negativos de los poderosos grupos económicos y políticos, el cabildeo y las actividades delictivas. También se omite a menudo la influencia de un contexto histórico y cultural de desigualdad y poder en la participación y el desarrollo. De todos modos, no hay que olvidar la dificultad que implica identificar, evaluar y comparar las fuentes y los efectos del capital social de manera generalmente aceptada y coherente en todos los ámbitos disciplinarios y los contextos sociales. 
Es particularmente interesante, en este marco, destacar entonces el trabajo realizado por Pisani et al. (2017), al proporcionar un método innovador para cuantificar y calificar el valor del capital social en los Grupos de Acción Local. El método se basa en indicadores teóricamente coherentes y construidos empíricamente. El método, de corte mixto (cuantitativo y cualitativo), se basa en un enfoque multidisciplinario para evaluar la naturaleza y el papel de la cooperación y la reciprocidad en el desarrollo local. A la vez, pretende capturar, integrar y medir las diversas formas de capital social y las maneras en que los actores interactúan, influyendo en las dinámicas de cooperación. El método es ahora una buena práctica sugerida por la Red Europea de Desarrollo Rural para los evaluadores de los programas de desarrollo rural, y se utiliza en las actividades regulares de monitoreo y autoevaluación llevadas a cabo por el GAL Prealpi y Dolomiti, en Italia (https://www.galprealpidolomiti.it/capitale-sociale/).

\section{Notas finales}

Esperamos que esta revisión de los avances investigativos en contexto europeo represente un aporte para el debate chileno. Evidentemente, este trabajo podrá ser complementado por otros que aborden las perspectivas latinoamericanas (y la chilena en particular), que sin duda se ha ido enriqueciendo de contribuciones muy relevantes en los últimos años.

En Chile diversos autores han estudiado este tópico; quizás el investigador más reconocido por su trayectoria es John Durston, activo colaborador de la CEPAL. Sin embargo, en la última década, investigadores del área social, económica, medioambiental y de las comunicaciones (que no mencionamos por tema de espacio), se han sumado a la tarea de profundizar en términos teóricos los contenidos de este concepto, y de aplicarlo a distintas realidades.

A nuestro modo de ver, particularmente interesante para el caso chileno parece ser el desafío de desarrollar un sistema de indicadores consolidado que permita profundizar el conocimiento sobre el capital social en una perspectiva aplicada. Un referente importante es sin duda el trabajo realizado con los GAL del programa Leader de la Unión Europea que acabamos de mencionar, pero existen también experiencias locales que se pueden recuperar, como la que desarrolló el PNUD para el Informe de Desarrollo Humano del año 2000 (Guell y Marquez, 2001).

Entre muchos, y solamente a partir de nuestro trabajo, visualizamos al menos estos tres espacios potenciales:

1) En el seguimiento y evaluación de políticas públicas y programas (implementados tanto a nivel rural como urbano) que tienen un enfoque territorial y consideran entre sus ejes de intervención el fortalecimiento de 
las comunidades locales; nos referimos en este caso a iniciativas como el Quiero Mi Barrio del MINVU, el Acción en Comunidad y el Más Territorio del FOSIS, entre otros;

2) En la evaluación del desarrollo de las cooperativas y del sistema cooperativo en general (relaciones entre socios, pero también entre cooperativas que conforman federaciones, confederaciones y/o asociaciones gremiales); en este caso se puede considerar el capital no solamente como un "medio" para el aumento de la capacidad de las cooperativas de generar ganancias para sus socios, sino también como un "producto" que fortalece el tejido socio-económico de un territorio dado; y

3) Finalmente, creemos que sin duda sería un aporte para el tercer sector chileno, conformado por fundaciones, ONG's y otros tipos de organizaciones, que históricamente han tenido la dificultad de medir el impacto de sus iniciativas sobre las comunidades que intervienen. Este trabajo no se agotaría en un ejercicio instrumental o en la mejora de la capacidad de rendición de cuentas de instituciones que en algunos casos han sido cuestionadas, sino también redundaría en el fortalecimiento de un conjunto de actores que desde el regreso a la democracia han vivido una crisis de identidad, y que podrían de esta manera reafirmar su importancia en los procesos de empoderamiento de las comunidades y del fortalecimiento de los tejidos socio-económicos locales.

Para cerrar el texto, nos queda solamente remarcar el espíritu que ha guiado este trabajo: desde la academia necesitamos avanzar hacia el desarrollo de investigaciones científicas que tengan un impacto en el entorno, y muy especialmente en aquellos contextos que tienden a ser marginados por las formas socio-económicas hegemónicas. En este sentido, profundizar los estudios sobre el rol del capital social en el desarrollo rural, se vuelve relevante para dotar a los decisores públicos de mejores herramientas, pero también para apoyar a las comunidades locales que tratan -en condiciones muchas veces adversas- de impulsar procesos participativos o de acción colectiva para mejorar su nivel de vida y el territorio en general. 


\section{Referencias}

Arrow, K. J. (1972). Gift and exchange. Philosophy and Public Affairs, 1, 343-362.

Bagnasco, A., Piselli, F., Pizzorno, A., y Trigilia, C. (2001). Il capitale sociale. Istruzione per I'uso. Bologna: II Mulino.

Becker, G.J. (1974a). Human Capital. New York: Columbia University Press.

Becker, G.J. (1974b). A theory of social interactions. Journal of Political Economy, 82(6), 1063-1093.

Becker, G.J. (1996). Accounting for tastes. Cambridge: Harvard University Press.

Becker, G.J. y Murphy, K.M. (2000). Social Environment. Cambridge, Harvard University Press

Bosworth, G., Rizzo, F., Marquardt, D., Strijker, D., Haartsen, T., y Aagaard Thuesen, A. (2016). Identifying social innovations in European local rural development initiatives. The European Journal of Social Science Research, 29(4), 442-461.

Bourdieu, P. (1980). Le capital social. Actes de la Recherche en Sciences Sociales, 31, 2-3.

Bourdieu, P. (1986). The forms of capital. En J.G. Richardson (Ed.), Handbook of theory and research in the sociology of education (pp. 241-258). New York: Greenwald.

Cecchi, C., Grando, S., y Sabatini, F. (2008). Campagne in sviluppo. Capitale sociale e comunità rurali in Europa. Torino: Rosenberg \& Sellier.

Coleman, J. S. (1988). Social capital in the creation of human capital. American Journal of Sociology, 94, 95-120.

Coleman, J. S. (1990). Foundations of social theory. Cambridge: Harvard University Press.

De Devitiis, B., Lopolito, A., Maietta, O.W., y Sisto, R. (2009). Innovazione e capitale sociale: il ruolo delle relazioni nelle imprese di trasformazione dei prodotti di agricoltura biologica. Rivista di Economia Agraria, 64(3-4), 291-318.

Durlauf, S. (2002). On the empirics of social capital. Economic Journal, 112(483), 459-479.

Durlauf, S., y Fafchamps, M. (2004). Social capital. NBER Working Paper nº 10485. 
Farrel, G., y Thirion, S. (2005). Social capital and rural development: from win-lose to win-win with the LEADER initiative. En D. Schmied, (Ed.), Winning and losing: the changing geography of europe's rural areas (pp. 45-61). Aldershot: Ashgate Publishing Ltd.

Fine, B. (2001). Social capital versus social theory: political economy and social science at the turn of the millennium. London: Routledge.

Franceschetti, G. (2009). Capitale sociale e sviluppo rurale. Le potenzialità dell'approccio LEADER e la sua trasferibilità. Padova: CLEUP.

Granovetter, M. (1973) The strength of weak ties. American Journal of Sociology, 78(6), 1360-1380.

Granovetter, M. (1985) Economic action and social structure: the problem of embeddedness. American Journal of Sociology, 91(3), 481-510.

Granovetter, M. (2018). The impact of social structure on economic outcomes. En M. Granovetter, y R. Swedberg (Eds.), The sociology of economic life. London: Routledge.

Guell, P., y Márquez, R. (2001). El capital social en el Informe de desarrollo humano en Chile 2000. En J. Durston y F. Miranda (Eds.), Capital social y políticas públicas en Chile. Santiago de Chile: CEPAL.

Hanifan, L. J. (1916). The rural school community centre. Annals of the American Academy of Political and Social Sciences, 67, 130-38.

Horlings, L., y Marsden, T. (2012). Exploring the new rural paradigm in Europe: eco-economic strategies as a counterforce to the global competitiveness agenda. European Urban and Regional Studies, 21(1), 4-20.

Koutsou, S., Ragkos, A., y Partalidou. M. (2014). Young farmers' social capital in Greece: trust levels and collective actions. Journal of Rural Studies, 34, 204-211.

Landry, R., Amara, N., y Lamari, M. (2002). Does social capital determine innovation? To what extent? Technological Forecasting and Social Change, 69(7), 681-701.

Lin, N. (1999). Building a network theory of social capital. Connections, 22(1), 28-51.

Lin, N. (2001). Social capital: a theory of social structure and Action. Cambridge: Cambridge University Press. 
Lin, N. (2003). Capitale sociale: paradigmi ricorrenti e loro validazione concettuale ed empiric. Inchiesta, 33, 5-17.

Lin, N. (2005). A network theory of social capital. Recuperado de: http://www. pro-classic.com/ethnicgv/SN/SC/paper-final-041605.pdf

Lin, N. (2017). Advancing network analysis of chinese businesses: commentary on Burt and Burzynska. Management and Organization Review, 13(2), 269-274.

Nardone, G., Sisto, R., y Lopolito, A. (2007a). II Capitale sociale nelle politiche di sviluppo rurale: I'esperienza dell'I.C. LEADER + in provincia di Foggia. Rivista di Economia Agraria, 1, 65-90.

Nardone, G., Sisto, R., y Lopolito, A. (2007b) Partenariato locale e capitale relazionale potenziale in provincia di Foggia. Rivista di Politica Agricola Internazionale, 1, 95-107.

Pisani, E., Franceschetti, G., Secco, L., y Christoforou, A. (2017). Social capital and local development. Basingstoke: Palgrave Editions.

Putnam, R.D., Leonardi, R., y Nanetti, R.Y. (1993). Making democracy work: civic traditions in modern Italy. Princeton: Princeton University Press.

Raagmaa, G. (2015). Social capital and rural development in the knowledge society. European Journal of Development Research, 28(2), 354-356.

Subramaniam, M., y Youndt, M. A. (2005). The influence of intellectual capital on the types of innovative capabilities. Academy of Management Journal, 48(3), 450-463.

Tamásy, C., y Revilla, J. (2016). Regional resilience, economy and society: globalising rural places. London: Routledge.

Teilmann, K. (2012). Measuring social capital accumulation in rural development. Journal of Rural Studies, 28(4), 458-465.

Vespasiano, F., y Martini, E. (2008). Innovazione senza capitale sociale. Pubblicazioni DASES Dipartimento di Analisi dei sistemi economici e sociali Università degli Studi del Sannio. Milano: Franco Angeli.

Welzel, C., Inglehart, R., y Deutsch, F. (2005). Social capital, voluntary associations and collective action: which aspects of social capital have the greatest 'civic' payoff? Journal of Civil Society, 1(2), 121-146.

Wiesinger, G. (2007) .The importance of social capital in rural development, networking and decision making in rural areas. Revue de Géographie Alpine, 95(4), 43-56. doi:10.4000/rga.354 
Wiesinger, G., Vihinen, H., y Tapio-Bistrom, M.L. (2005). The role of social capital in rural development. Conclusions from a European project on marginalisation and multifunctional landuse. Paper submitted to the XXI ESRS Congress Keszthely, Hungary 22-27 August 2005.

Woolcock, M., y Narayan, D. (2000). Social capital: implications for development theory, research, and policy. The World Bank Research Observer, 15(2), 225-249.

Dirección de correspondencia:

Stefano Micheletti

Magíster en Cooperación para el Desarrollo y Responsabilidad Social y Ambiental.

Académico del Centro de Estudios Urbanos-Territoriales de la Universidad Católica del Maule, Talca, Chile.

Contacto: smicheletti@ucm.cl 